\title{
Journalistes et communicateurs au temps de
} l'information existentielle

\section{François Demers}

\section{(2) OpenEdition}

1 Journals

Édition électronique

URL : http://journals.openedition.org/communicationorganisation/1805

DOI : 10.4000/communicationorganisation. 1805

ISSN : $1775-3546$

Éditeur

Presses universitaires de Bordeaux

\section{Édition imprimée}

Date de publication : 1 novembre 1995

ISSN : 1168-5549

Référence électronique

François Demers, « Journalistes et communicateurs au temps de l'information existentielle », Communication et organisation [En ligne], 8| 1995, mis en ligne le 26 mars 2012, consulté le 03 mai 2019. URL : http://journals.openedition.org/communicationorganisation/1805; DOI : 10.4000/ communicationorganisation. 1805

Ce document a été généré automatiquement le 3 mai 2019.

(c) Presses universitaires de Bordeaux 


\title{
Journalistes et communicateurs au temps de l'information existentielle
}

\author{
François Demers
}

1 Dans les pays développés, l'information journalistique des années 90 offre un visage qui, par comparaison avec celui qu'elle présentait dans les années 70, fait davantage penser à la rupture qu'à la continuité. Ce qui frappe en premier lieu, c'est sa fragmentation, à la mesure même de la multiplication des lieux qui l'offrent: quotidiens, magazines, et chaînes de télévision. Mais il y a aussi cette domination évidente de la télévision, et plus généralement encore de l'image dans le concert des médias: omniprésence des graphiques, de la couleur et des photos dans la presse écrite, direct et effets visuels dans l'électronique. On constate par ailleurs l'importance croissante, en termes de ressources, d'espace alloué et de temps d'antenne, de domaines plus triviaux et directement utilitaires, tels la météo, la bourse, l'état des routes et de la circulation, les tests et discussions autour des produits et services de consommation courante (arts, sports, autos, etc.) ainsi que les reportages « humains » en général. Enfin, l'émotion est devenue omniprésente: par les sujets traités, violents et dramatiques, par l'attitude des interviewés saisis dans des moments-chocs autant que, de plus en plus, par l'implication émotionnelle des animateurs et des reporters.

2 L'une des explications globalisantes du changement repose sur le mercantilisme des médias et leur soumission de plus en plus débridée, dans le cadre d'une concurrence exacerbée, au marketing comme loi d'airain de la satisfaction du public à tout prix : l'information s'alignerait de plus en plus sur ce que le public veut savoir plutôt que sur ce que le public doit savoir.

3 Cette perception et critique est ancienne, quasi contemporaine de la naissance de la presse de masse. Dès le début en effet, les milieux conservateurs ont attiré l'attention sur le danger d'un alignement du contenu sur les « goûts dépravés de la classe ouvrière ». De leur point de vue, qui s'est exprimé sous différentes versions depuis, «les tentatives de faire appel à un public de masse au moyen d'un discours rationnel étaient destinées à 
échouer, les masses n'étant attirées que par des sujets élémentaires et émotionnels » (Murdock, 1982 : 91).

Dans cette version des choses, ce qui se passe aujourd'hui se présente nettement comme une dépravation: du haut de la culture de l'élite, on s'efforce d'attirer l'attention sur la violence, la vulgarité et le sensationnalisme des reportages et des shows journalistiques.

Une autre explication découle de la comparaison entre les pratiques du réseau d'information scientifique et technique et celles du réseau dit de "l'information existentielle", selon l'expression de Jacques Ellul (1990: 346). Le premier est fondé sur des exigences de mise à distance des émotions et d'univocité des termes. Le second, lui, est de «l'ordre des informations de la vie courante, de l'opinion, des sentiments, des idées, etc.» Evidemment, l'information scientifique sert d'étalon condamnant la « confusion extrême de l'information » dans l'existentiel. D'ailleurs, Ellul estime que les deux réseaux s'éloignent de plus en plus l'un de l'autre. Mais le jugement qu'il porte est ambigu parce qu'il salue d'autre part la polysémie et l'ambiguïté «merveilleuse » du langage de la vie quotidienne qui «permet justement la richesse et la multiplicité des relations humaines, la possibilité d'évolution et d'institution des cultures. Sans flou de l'information, aucune culture n'est possible » $(1990: 346)$.

6 Il reste que cette thèse vise la superficialité des analyses et des commentaires journalistiques, leur manque de mémoire historique et plus généralement, leur trop faible culture. Elle est particulièrement choquée par l'idiote télévision.

7 L'article qui suit renverse en quelque sorte ces perspectives puisqu'il propose d'interpréter les phénomènes en cause comme une libération de l'information existentielle face à la contrainte qu'imposait le précédent ordre de l'information. Empruntant l'éclairage du postmodernisme, il suit le fil conducteur des considérations de Jean-François Thuot qui, dans un article récent sur les formes postmodernes de la démocratie, rappelle que l'État libéral s'inscrivait au départ « dans une logique de mise en tutelle des pratiques particulières des acteurs sociaux" au nom "d'un ordre social désirable, d'un ordre social à édifier, incarné par l'idéal républicain » (Thuot, 1994 : 77).

Thuot fait l'hypothèse que cette légitimité de l'État à transcender le peuple réel est sur son déclin et que le politique est désormais sommé « de faire corps avec la société ». Nous serions passé de la démocratie représentative à la démocratie fonctionnelle, laquelle participe d'un mouvement de "dissolution de la norme dans le fait». Or l'information journalistique, cette partie plus ou moins volumineuse du contenu des médias de masse entre la publicité et les variétés, est précisément née dans le giron de l'État libéral. Ce qui autorise à penser que sa trajectoire risque fort d'être identique à celle de ce dernier. L'exploration de cette avenue comporte ici deux parties: la première va démarrer avec l'affirmation que, dès le départ, informer signifiait imposer une relation hiérarchique et autoritaire entre producteurs et récepteurs. C'est la communication qui s'est faite l'étendard de la contestation de cette autorité et de l'arbitraire. Elle s'est affirmée comme exigence de circulation des messages des autres acteurs sociaux et comme refus de la verticalité. La seconde partie va mettre en scène, elle, trois " changements structuraux » que l'on dit constitutifs de la rupture postmoderne, laquelle assurerait en quelque sorte le triomphe et la revanche des destinataires des messages, de leur réalité différenciée et fragmentée. Ce sont « le déclin de l'efficacité de l'Etat-nation moderne (à l'intérieur de ses frontières autant qu'à l'extérieur), les transformations économiques dans les procès de production et l'organisation des entreprises, ainsi que la réorientation de la culture en direction de la consommation mass-médiatisée » (Dickens \& Fontana, $1994: 11$ ). 
9 Mais qu'arrive-t-il des professionnels de l'information, les journalistes et les communicateurs (quelquefois nommés relationnistes), eux dont le métier a pris forme dans un contexte du pouvoir concentré et transcendant ? Cela fera l'objet de la conclusion alors qu'il sera affirmé que leur relation de compétition/collaboration (Charron, 1991) est désormais subordonnée à l'impératif général de séduction qui oriente sur la place publique l'action des groupes de pressions et minorités diverses, aussi bien que celle des représentants politiques. C'est la séduction qui permet la construction de coalitions majoritaires, fragiles, temporaires et mouvantes, "selon une logique contractuelle et multipolaire » (Thuot, 1994 :100).

\section{La communication contre l'information}

10 La courte histoire de l'information journalistique s'étend sur à peine un peu plus d'un siècle. Elle prend naissance au sortir de la presse d'opinion alors que, forts des libertés bourgeoises récemment conquises, les entrepreneurs politiques et religieux multiplient les feuilles chargées de diffuser au sein des élites instruites leurs opinions sur les façons rationnelles de gérer les affaires collectives. Puis quelques-uns d'entre eux inventent le moyen de faire de l'argent avec un produit que l'on nommera l'information. Ils se transforment en entrepreneurs de presse (de Bonville, 1988).

11 L'information comme substantif désignant les exposés d'opinion et de narration à propos des faits de sociétés et des événements, prend corps peu à peu. Elle finit par s'affirmer dans toute sa force au début de ce siècle alors que la presse quotidienne court vers le monopole ou le quasi-monopole dans la plupart des grandes villes. Naissent alors les médias de masse, quotidiens dans les grandes concentrations urbaines, magazines mensuels à l'échelle nationale.

12 Un énorme pouvoir de magistère et de prêche est ainsi concentré entre les mains de quelques haut-parleurs devenus fourches caudines pour les acteurs sociaux autres que les entrepreneurs de presse et leurs plumitifs à gages, les journalistes. Le média-entreprise obéit à sa logique propre : pour lui, le reste de la société est un vivier dans lequel il puisse les matériaux des histoires qu'il raconte à son propre profit d'abord. Au départ de ce cycle, propriétaires de presse et journalistes profitent de leur rôle d'intermédiaires obligés et de détenteurs de l'unique chaire de la place publique. Cela devint vite trop manifeste au temps de la phase débridée du journalisme populaire, de ses campagnes de presse, de son "journalisme jaune" et de son «muckraking", l'ancêtre du noble journalisme d'enquête. Puis, peu à peu, la position de force des seigneurs de la presse s'est effritée, en partie parce que des moyens techniques ont été inventés, assemblés et gérés de façon à offrir aux autres acteurs sociaux et économiques des alternatives, mais aussi parce qu'ils ont dû composer avec les autres acteurs sociaux qui se sont efforcés de diverses façons de harnacher leur pouvoir disrupteur.

13 Cela explique notamment les si nombreuses études sur le « gatekeeping » et le " agendasetting » (Charron, 1995) préoccupées d'identifier les mécanismes de la sélection et de la construction de l'information pour mieux les discréditer, les contourner ou les manipuler. C'est aussi dans ce contexte qu'est né l'agent de presse chargé d'influencer médias et journalistes en faveur de son employeur. Souvent ex-journalistes, les agents de presse, bientôt rebaptisés relationnistes puis communicateurs, sont d'entrée de jeu des challengers des journalistes avec lesquels ils pouvaient rivaliser dans la maîtrise 
artisanale du langage grand public (Grunig \& Hunt, 1984). Témoignent de leur succès les innombrables communiqués de presse qui sont aujourd'hui diffusés par les médias presque sans retouches (FPJQ, 1988).

Bref, dénonçant les biais, les manipulations et les distorsions, « les autres » exigeaient que les médias se fassent davantage transparents et que recule l'arbitraire. Ils en sont venus à revendiquer contre l'information des propriétaires de presse et des journalistes, une communication, définie essentiellement comme circulation des messages entre les publics, les groupes de pression, les partis politiques, les individus et les entreprises. Finalement, les journalistes durent restreindre leur pouvoir d'opinion, faire place et transmettre fidèlement les messages des autres acteurs sociaux. Après avoir désigné le récit des faits de société tels que vus et arrangés par les gens de presse, le concept d'information en vient à désigner l'espace médiatique débarrassé des opinions des journalistes et des propriétaires des médias, désormais refoulées dans l'espace éditorial. L'information se légitime alors en s'affirmant véhicule « objectif» des points de vue des personnalités et des groupes de pression. Elle se fait vecteur des messages des autres. Jusqu'au jour où quelqu'un comme Dominique Wolton (1989) pourra conclure, pour dénoncer ce fait, que l'information est devenue communication.

En parallèle, à partir d'horizons plus radicaux- le socialisme, la contre-culture, le féminisme-, les critiques concluent au blocage pur et simple des médias de masse face à certaines idées et réalités. Ils prescrivent dès lors qu'on les contourne et les double. Pour certains, le refus global trouve son origine dans le constat pragmatique que leurs causes et leurs opinions sont carrément indigestes pour l'information et qu'elles ne parviennent pas à y trouver place ou ç y être traitées de façon adéquate. Pour d'autres, ce qui importe, c'est la relation radicalement inégale entre des émetteurs rares et une masse de récepteurs atomisés. Tout ce monde se résume autour d'un idéal de communication comme dialogue. L'enjeu deviendra de multiplier le nombre de lieux de production et à la limite de transformer chaque consommateur de messages en producteur de messages. De la presse alternative à la télévision communautaire, en passant par la radio populaire, jusqu'à la télématique et à Internet, on se rallie en brandissant l'étendard de la communication horizontale (Wasco et Mosco, 1992).

\section{Du spectacle à la conversation}

16 Jusqu'à l'affirmation de la télévision comme média dominant du système massmédiatique dans son entier, la relation de l'information avec son public était caractérisée, outre par sa verticalité, par la distance psychologique. Malgré leurs effets de style et leurs appels à l'imagination, la presse et la radio - des médias « chauds » au sens de McLuhan, "plongent en effet leurs destinataires dans un état de réceptivité passive» et entretiennent «la mentalité du consommateur» (McLuhan, 1977: 12). Cette distance constitutive est identique à celle du spectateur face à une cérémonie, à une pièce de théâtre et généralement à tout ce se passe devant lui, avec un regard dans sa direction, mais sans lui. L'investissement émotif reste faible et la distance constante.

C'est la télévision qui a changé les règles de ce jeu. Elle s'est d'abord inscrite, au rang de la presse et de la radio, comme fournisseur de spectacles, un spectacle plus poignant au départ parce que plus cinématographique. C'était compter sans l'effet de saturation, la démystification et la banalisation qui a suivi son omniprésence. La relation avec elle, devenue objet familier, s'est peu à peu transformée en une sorte de conversation (Deli 
Carpini et Williams, 1994). Comme les conversations de la vie quotidienne, le dialogue offert par la télévision offre des moments de plus ou moins grande intensité. De même, il fusionne contenus cognitifs et contenus émotionnels. Comme dans la vraie vie, ce qui compte le plus souvent dans cette conversation entre télévision et téléspectateur, c'est moins le contenu de l'information comme référent externe que la pseudo-personne qui en parle, c'est-à-dire le journaliste, le présentateur, la chaine en tant que personnalité. Le consommateur peut ainsi réagir au contenu et du même souffle au messager qui le raconte, dans une globalité semblable à la vie quotidienne, avec $90 \%$ du temps la même banalité répétitive et avec, parfois, des moments spectaculaires et intenses, parce qu'une masse y communie.

18 Comme pour les conversations de la vraie vie, la pseudo-conversation avec la télévision, qui occupe 4 à 5 heures de la vie quotidienne des Nord-Américains, est le plus souvent inutile, parce qu'elle n'est pas vraiment «un intermédiaire par rapport à un monde ambiant, au milieu naturel ou micro social» (Ellul, 1990 : 337). Ou plutôt, elle est utile dans les trois sens restreints suivants, en insistant sur le troisième, celui où il est question de passer le temps en ressentant des émotions sans conséquences.

19 1) l'utilité de cuisine offre des renseignements pour la conduite de la vie pratique, des recettes, des données pour les placements financiers, des arguments pour les militants, des éléments d'information technique, etc.

2) avec l'utilité de chambre à coucher, le bénéfice se prend pendant la consommation ellemême. Cela veut dire que le reportage est intéressant pendant qu'on le regarde ou qu'on le lit : c'est bien raconté, c'est bien écrit.

Au sortir, peu importe qu'il n'en reste rien, il y a eu plaisir à consommer.

3) l'utilité de salon, c'est l'information qui sert à la vie publique. Se ternir au courant permet de meubler les conversations avec ses voisins et les gens de son réseau social, si réduit soit-il. L'information sert le plus souvent du même fait à renseigner le citoyen en fonction de ses activités politiques.

L'information de salon, dans son volet qui vise le citoyen-électeur, est davantage de type cognitif. Elle constitue le visage noble de l'information journalistique et à ce titre, elle est la plus valorisée par les analystes et commentateurs. C'est elle que l'on tente de juger à l'aulne de l'information scientifique et technique, ou encore dont on jauge la valeur culturelle en ayant en tête la culture d'élite. L'information de cuisine, elle, est nettement plus prosaïque : elle va à la rencontre, plus ou moins en aveugle, des innombrables besoins de la vie quotidienne : un nom, une date, un numéro de téléphone, une recette, etc. Elle ne se réalise que lorsque tel ou tel consommateur trouve accidentellement quelque chose qui correspond à ses besoins du moment. Elle suppose un consommateur qui parcourt des quantités énormes d'information, l'attention minimalement en éveil, minimalement parce que l'information est généralement consommée en période de loisirs ou en marge d'autres activités; elle est associée à la détente et non à l'effort. L'utilité de chambre à coucher, elle, occupe les temps libres avec des émotions ; comme gratuitement.

Aujourd'hui, le concept de communication recouvre, sédimentées, ces trois préoccupations à la fois successives et parallèles: circulation des opinions, recherche d'un rapport plus interactif entre émetteur et récepteur, messages plus humains et holistes, autant émotifs que cognitifs. En face, l'information résiste. Forte de la liberté de 
presse et du droit de propriété de l'entrepreneur, elle continue d'affirmer son droit au magistère, au choix de ce qui peut circuler et à la critique des autres acteurs sociaux.

\section{Le repli de l'Etat-nation} «multinationales» ou "mondiales» des industries culturelles. Celles-ci percent ou sautent les frontières et les protectionnismes par des moyens techniques: Internet, les satellites, les cassettes et disquettes, etc, par les alliances avec des firmes autochtones, par l'intégration des ex-médias nationaux privés dans des chaînes et des empires ou tout simplement par la constitution de médias à intention mondiale tel CNN. Elles seraient porteuses d'une culture mondiale, certainement occidentale, sinon américaine. De fait, les plus grands conglomérats médiatiques sont d'origine états-unienne et les firmes d'autres enracinements nationaux - dont les canadiennes - se livrent à diverses manœuvres pour grossir jusqu'à pouvoir les affronter dans l'arène mondiale. Ainsi, au-dessus des frontières, se dessine une nouvelle centralité en construction, un nouveau lieu d'autorité culturelle et de fabrication des représentations symboliques communes.

29 Le repli de l'Etat-nation signifie aussi une dénationalisation plus ou moins rapide et exhaustive de la place publique. Les frontières s'ouvrent aux médias étrangers. Les produits étrangers offrent une version autre des activités nationales, plus ou moins divergente du regard proposé par les médias du cru. Lorsque l'écart est grand, l'effet peut 
être fortement déstabilisateur. D'autre part, l'État déréglemente l'industrie nationale, laissant libre cours au foisonnement des nouvelles entreprises, aiguillonnant d'autant la concurrence et précarisant tout le monde sauf les plus gros. En même temps, il coupe les vivres aux médias publics.

Tout cela crée un vide d'autant plus désorientant que l'action de l'État à travers ses médias, autant que par la législation et la réglementation, fixait comme norme générale de comportement le service public, le soutien au développement national et le protectionnisme culturel. Jusque-là, plus ou moins subtilement guidés par l'Etat-nation, les journalistes et leurs jumeaux communicateurs obéissaient les uns et les autres à un programme de surveillance, de traitement et de création des événements où le politique, en tant que lieu de gestion des affaires collectives, apparaissait central et prioritaire. Cette assignation était conforme à la doctrine des Lumières qui assignaient aux médias une mission en lien direct avec les activités de l'État. La dé-nationalisation entraîne donc aussi la perte des effets structurants du politique sur le territoire de l'information.

\section{Fragmentation et différentiation}

31 Le desserrement du contrôle des Etats-nations se conjugue à de nouvelles possibilités techniques pour favoriser la multiplication des médias et l'abondance de l'offre. Celle-ci stimule la concurrence qui, à son tour, libère le processus de différentiation médiatique, lequel renverse la relation autoritaire de l'information au profit des différences venues d'en-bas dont se saisissent l'entreprenariat et l'écoute marketing. Ceux-ci instaurent alors un «fétichisme du choix personnel» et engendrent «un important mouvement d'intolérance à l'égard de l'unicité, de la rigidité et de toute forme d'autorité qui persiste à vouloir imposer à tout prix ses valeurs. L'hétérogénéité et le pluralisme deviennent des valeurs de plus en plus défendues à l'intérieur de nos sociétés postmodernes » (Boisvert, 1995 : 30).

Ainsi, à l'intérieur des territoires des Etats-nations envahis par l'étranger, la scène médiatique éclate aux niveaux local et régional en morceaux centrifuges qui multiplient l'offre de produits. À niveau local, quasi chacun peut sortir son journal, se doter d'un émetteur radio ou d'un équipement de production vidéo. Ce phénomène s'inscrit dans la résurgence générale des particularismes locaux, culturels, linguistiques et ethniques. Tout se passe comme si bondissaient à l'avant-scène des forces et des valeurs longtemps nivelées, combattues, contraintes par les appareils d'État à l'intérieur des cadres nationaux. Paradoxalement, comme l'indiquent Tracey et Redal (1995) à propos de la production télévisuelle, les particularismes sont si puissants que, sous le visage du "provincialisme», ils arrivent à faire reculer les produits offerts par les réseaux internationaux. Il y a d'ailleurs une prévisible correspondance entre la popularité des productions autochtones et l'importance de l'information dite «de proximité » dans les investissements traditionnels des salles de rédaction. L'État national, volontaire, interventionniste et musclé faisait manifestement écran.

33 À la fin, taillée en pièces, la place publique unique et centrale, tracée hier par les médias de masse nationaux, publics et privés, disparaît peu à peu au fur et à mesure que ceux-ci sont remplacés par les médias sectoriels et spécialisés, locaux, micro-locaux et transnationaux. Dans ce contexte, les minorités peuvent mieux s'identifier elles-mêmes, se regrouper autour de «leurs » médias, elles peuvent mieux se parler à elles-mêmes, leurs membres peuvent mieux se parler entre eux. Mais il y a de moins en moins de place 
commune où elles peuvent dialoguer. Le principal, sinon l'unique territoire d'intersection médiatique commun à tous est aujourd'hui formé des messages multimédias usinés par les firmes multinationales de la publicité et par les boîtes de spécialistes de la communication au service d'institutions et d'entreprises qui œuvrent à niveau mondial.

La fragmentation des publics qui correspond à cette situation prolonge le processus de «différenciation des masses» auquel se réfère Laponce (1986: 217). L'affirmation des différences entre sous-groupes se révèle constitutive de la postmodernité dans la mesure où elle fonde la coexistence, sur un même pied de légitimité, de systèmes normatifs mutuellement exclusifs. À ce moment-ci du mouvement du balancier, les médias contribuent au relativisme et au chaos comme, à l'autre, celui de la consommation massifiée du temps jadis (et aujourd'hui encore à niveau de mondialisation), ils fabriquaient l'homogénéité. Ou plutôt " contrairement à ce que prévoyaient plusieurs tenants de la modernité, le développement des Communications et des échanges d'informations n'a pas conduit nos sociétés à une transparence totale ni à l'universalisation. Selon [les postmodernistes], il a plutôt engendré un accroissement des prises de parole, entrainant la mise en place de sociétés plus complexes, voire chaotiques. Les médias, purs produits de la modernité et de la culture moderne, n'ont donc pas œuvré à l'universalisation comme l'on s'y attendait, mais ont plutôt favorisé le pluralisme » (Boisvert, 1995 : 34, 35).

Car l'abondance de l'après-guerre, dans les pays industrialisés, a multiplié les entreprises de presse et exacerbé la concurrence, faisant basculer la perspective des entrepreneurs dans un impératif de rejoindre, séduire et représenter un public-cible. De telle sorte que ce déverrouillage du marché des idées implique à terme la dérive vers la rhétorique, c'està-dire vers les opérations incessantes de séduction par les acteurs de l'actualité, dont les médias eux-mêmes.

On assiste donc à une floraison des news-letter, bulletins de liaison et publications de courte série en raison des possibilités de l'édition informatisée. Le nombre des chaînes de télévision accessibles à faible coût explose par suite de la multiplication des satellites et l'extension des réseaux câblés. Cette fragmentation des marchés entraîne, autant qu'elle est entraînée par elle, l'exacerbation de la concurrence. Celle-ci stimule la fragmentation et la différentiation des publics aussi bien que celles de l'offre. Mais la multiplication de l'offre n'exclut pas à un autre niveau la concentration de la propriété sous forme de conglomérats, de holdings et d'empires divers, nationaux et transnationaux.

Le passage de la rareté relative à l'abondance érode le pouvoir des médias et des journalistes. Le goulot d'étranglement médiatique se distend offrant désormais pratiquement à toutes les causes un canal pour se faire entendre. Par contre, si tout un chacun peut espérer accéder à un haut-parleur, il est loin d'être assuré qu'il y aura masse pour l'entendre. Car la multiplication de l'offre signifie aussi l'augmentation des choix possibles du côté des consommateurs. La réorganisation de la production a entraîné la fragmentation de l'offre, le règne du choix, la pratique généralisée du "zapping", l'infidélité du consommateur, le bricolage du sens des messages par le récepteur, jusqu'à la communication horizontale et hyperpersonnalisée de l'Internet.

Mais la concurrence a des effets ambivalents aussi bien en matière politique que commerciale : l'entrepreneur est poussé à souligner ses différences pour construire son premier marché, fractionnant ainsi d'autant le marché général. Très vite, cependant, s'il ne veut pas être enfermé dans ce premier rassemblement autour de lui, il doit se lancer 
dans des opérations de charme pour gruger le marché des autres et élargir d'autant le groupe de ses fans ou clients, jusqu'au monopole. D'oü ses incessants efforts de séduction.

\section{Le règne du marketing}

Au cours des années 80 , la fragmentation des publics et la multiplication des supports ont provoqué la panique chez les grands médias de masse traditionnels. La menace de perdre le monopole des messages payants, c'est-à-dire la publicité, les a poussé à réagir en soumettant encore davantage et d'une manière plus directe l'information aux réclames dans le cadre des médias gratuits, des publi-reportages et des talk-shows. Mais ces mesures auront été insuffisantes. Depuis la fin des années 80 , une partie significative des budgets publicitaires fuit vers d'autres supports que les médias traditionnels (Business Week, 1991). Il devient de moins en moins coûteux et de plus en plus techniquement facile aux groupes et firmes de se projeter sur la place publique directement, dans un média qu'ils contrôlent entièrement: feuillets ou dépliants, bulletins de liaison, Internet, infomerciaux et chaînes publicitaires (Bédard, 1995). La publicité pousse elle aussi à la fragmentation.

Pour les médias de masse, c'est la, dans le desserrement de l'alliance entre publicité et l'information, que le changement blesse le plus. En effet, c'est la cohabitation entre ces deux types de messages qui avait fondé la presse d'information en assurant la gratuité ou la quasi-gratuité de l'information journalistique pour le consommateur-citoyen. Cette alliance historique avait permis aux entreprises de presse de se placer au centre du jeu, en position de goulot d'étranglement dans les circuits de la communication publique. Dans les années récentes, la presse écrite a été forcée de compter de plus en plus sur l'abonnement et la vente au numéro pour se financer et payer le coût de la production de l'information journalistique. D'autre part, le paiement à l'usage, central dans les stratégies des câblo-distributeurs et des distributeurs par satellites, annonce la séparation du couple en ce qui concerne la télévision. Des chaines de télévision spécialisées et des bulletins de liaison électroniques se libèrent en effet de la publicité. Les produits sans pub peuvent se multiplier au fur et à mesure que la tarification à l'usage devient techniquement facile et peu coûteuse, quitte à ce que ces oasis ne soient accessibles qu'à des membres de "clubs privés ", capables de payer des cotisations substantielles, selon l'image forte développée par Tremblay et Lacroix (1991).

41 Cela pose des problèmes radicaux de stratégie pour les émetteurs mus par la volonté d'influencer. Il faut recomposer ailleurs l'unicité du discours émis. D'un côté, il faut se donner les moyens d'accéder aux principaux médias et réseaux transnationaux et concevoir des campagnes publicitaires transnationales, par exemple, celles de Benetton qui mise sur la sensibilité adolescente des jeunes instruits de tous les pays: messages provocateurs (aux yeux de leurs parents) en même temps que généreux (au-delà des mesquineries raciales ou religieuses). C'est aussi dans cette logique que l'on voit émerger des causes internationales - et des actions spectaculaires à ce niveau, telles celle des autochtones (Chiapas, protestations Mohawks contre le développement hydroélectrique du Nord québécois, etc.) ou celle de l'environnement avec Greenpeace (Dacheux, 1994).

En matière de journalisme, la concurrence veut dire dans bien des cas la spécialisation des contenus. Cela veut dire aussi l'écoute du marché : chercher ce que le public veut savoir alors que la tradition du journalisme met plutôt l'accent sur ce que le citoyen doit savoir. Ceci veut dire enfin, pour le journaliste-employé, une solidarité enthousiaste avec 
l'entreprise qui l'emploie et une identification la plus spontanée possible avec son public. Pour le journaliste préoccupé de promouvoir une vision du monde, d'influencer, d'éduquer, d'intervenir, il s'agit de trois réorientations déchirantes. Il ne peut plus invoquer une abstraction, tel le service public ou l'intérêt public, pour résister aux demandes du public réel ou à celles de son employeur.

Pour le relationniste, la nouvelle configuration de l'industrie demande un raffinement des méthodes. Il faut développer les moyens de connaître plus précisément les publics, leurs attentes, leurs bricolages et leurs besoins. Il faut complexifier les plans d'action: y introduire plus de supports, diversifier les messages, intégrer des étapes et des évaluations, etc. Il faut aussi dépenser plus d'énergie à concevoir les messages dont il faut d'ailleurs fabriquer plusieurs versions.

Bref, le repli plus ou moins accentué de l'Etat-nation a déboussolé les systèmes collectifs de communication de masse construits autour de frontières physiques et symboliques et centré sur les institutions politiques nationales. Pour les journalistes, cela pose le défi de l'éclatement des normes de la sélection de l'information : la hiérarchie qui accordait la priorité au politique et au social s'est en quelque sorte liquéfiée, plus ou moins selon la vigueur résiduelle des institutions et des représentations nationales. Dans de nombreux cas, les salles de rédaction continuent d'exercer leur liberté éditoriale sur la base des habitudes du passé. Mais les plus agressives se réalignent ouvertement sur les études de marché, c'est-à-dire sur les goûts, intérêts, besoins et sensibilités de leur public-cible. Domine le marketing, dans sa préoccupation que le produit ne soit défini qu'après qu'on ait bien pris le pouls des clients potentiels. La relation autoritaire, caractéristique du temps où dominait l'information, semble cassée. Les médias, comme les institutions politiques s'alignent sur la fonctionnalité : ils représentent plutôt qu'ils arbitrent. La norme se rapproche du fait.

Les relationnistes perdent eux aussi le Nord. Fondamentalement chargés de "gérer les enjeux sociaux » (Bartha, 1990), ils devaient déployer messages et gestes stratégiques de manière à influencer, intercepter ou neutraliser l'opinion publique et, à travers elle, les institutions publiques. Mais si celles-ci se retirent d'elles-mêmes ou sont discréditées, à quelles fins travailler l'image publique? Il ne reste que la subordination totale de la communication aux objectifs commerciaux à court terme et l'intégration des relations publiques dans le cocktail des actions de promotion en vue de la vente. C'est ainsi que dans les stratégies des firmes, l'intervention auprès des médias et de leurs journalistes devient moins prioritaire et s'inscrit parmi une série d'autres actions de communication possibles.

\section{Le domaine de l'existentiel}

Le repli de l'Etat-nation découle, autant qu'il le provoque, de l'effondrement dans l'ordre des idées, des symboles, des conceptions et des rêves, de la croyance en l'efficacité du politique, et plus généralement de la Raison et de la volonté collective, comme outil de progrès. En conséquence, les médias sont peu à peu libérés de l'exigence du politique : l'information n'est plus définie comme une activité indispensable à la gestion politique de la cité. Puisqu'il n'y a plus de point de vue transcendant que les autorités politiques et les médias pourraient imposer de force, il leur faut être constamment en marketing, rassembler, construire et reconstruire constamment, des coalitions fragiles. Vu des groupes de pression, ce qui importe, c'est la capacité de séduction, l'habileté à développer 
une rhétorique capable de rassembler des alliés et de former des majorités à des fins politiques ou économiques.

L'information devient un territoire où la hiérarchie des contenus et des priorités est déterminée par le marché. On y va à la rencontre des majorités lesquelles imposent leur préoccupation de l'information existentielle et leur basse culture, populaire. Evidemment, en s'alignant sur les préoccupations de la vie quotidienne, l'information dans une société de consommation ne peut éviter de tourner autour de questions de consommation et que se multiplient les publi-reportages. Tout comme, dans une télévision qui offre tout à la fois connaissance et émotion, il est inévitable que l'information prenne couleur de divertissement et que se généralise les pratiques d'infotainment (contraction des mots : information et entertainment).

Si on additionne le décrochage du politique, la spécialisation des contenus et des traitements, avec l'alignement sur les goûts du public, cela donne une situation où émergent comme prioritaires et légitimes: -les renseignements pratiques pour la conduite de la vie quotidienne ; - la météo et la circulation automobile pour la conduite dans l'environnement physique; - les cours de la bourse ou les essais de nouvelles voitures pour la conduite dans l'environnement économique ; - les nouvelles locales ou la vie des gens riches et célèbres pour la conduite dans l'environnement social, etc.

49 Mais les métiers de relationniste et de journaliste ont été mis au point dans un contexte où le politique occupait le centre de la légitimité de l'information: la liberté de l'information et la noblesse de la fonction de journaliste se fondaient sur sa fonction politique. Les autres domaines de l'information s'organisaient ensuite dans un ordre hiérarchique s'éloignant graduellement de ce centre, passant par les réalités sociales et économiques susceptibles de traduction politique, puis les arts de la haute culture, puis le sport, puis les arts populaires, jusqu'au sang, au sexe et aux potins.

50 La perte de ce point de référence s'additionne à la spécialisation des médias pour désarticuler ce qui était désigné comme l'Actualité, la même pour tous. Celle-ci était un construit sur la base à la fois d'une conception commune et de mécanismes exerçant des pressions centripètes. Le consensus accordait priorité aux institutions politiques et aux réalités sociales susceptibles d'être traduites politiquement. Les mécanismes, eux, par exemple le secrétariat de rédaction et les affectations fixes auprès de lieux institutionnels, assuraient la surveillance mutuelle entre les médias et les nécessaires effets d'alignement, de suivi et de relance. La désarticulation de l'Actualité libère elle aussi la différentiation des centres d'intérêts et facilite le jeu de la spécialisation des médias.

51 De la même façon, les fonctions répressives du " gatekeeping » et de «l'agenda-setting " ne peuvent plus être exercées au nom d'une Actualité qui serait la même pour tous les journalistes et dont le centre se trouverait dans la vie des organismes de l'État. Elles ne peuvent plus s'aligner que sur un public particulier que le média prétend représenter et desservir et qu'il fait s'exprimer à travers sondages, concours et analyses de tirage ou de cote d'écoute. Elles ne peuvent plus se légitimer qu'au nom de la liberté d'expression démocratique comme liberté d'entreprise politique ou religieuse ou commerciale.

52 Autre aspect du changement, les médias et les journalistes perdent leur mission éducative : il n'y a plus de corpus transcendant. Ni l'État, ni aucun autre acteur social ne peut aujourd'hui se dire porteur du point de vue de la Raison, transcendant les intérêts particuliers et les versions partielles et partiales. Dès lors, ne pouvant plus adopter un 
point de vue au-dessus de la mêlée des intérêts, le journaliste justifie son existence et sa volonté de puissance comme fabricant du sens : - soit comme entrepreneur, partisan de certaines thèses et/ou fabricant d'un produit qui cherche à plaire, c'est-a-dire comme éditeur-propriétaire d'un média dans le libre marché des biens et des idées ; - soit comme salarié d'une entreprise qui, elle, a quelque chose à promouvoir ou à vendre et qui utilise les acteurs-sources comme matériaux des contenus qu'elle apprête pour la vente, c'est-adire comme reporter. Dans ce cas, il doit participer émotionnellement à cette entreprise, devenir un «bon employé » et un médiateur entre le public et l'émotion que lui provoque l'événement (Lavoinne, 1992: 50). Il ne s'agit cependant plus de la médiation que l'on attendait du reporter « intermédiaire entre des sources et un public » (Ruelland, 1993 : 134 et 135). Cette conception insistait sur le rôle d'intermédiaire fidèle entre les sources / acteurs de l'actualité et le public. La même expression utilisée aujourd'hui attire plutôt l'attention sur le rôle d'intermédiaire entre le public et l'actualité, l'essentiel étant que le reporter-médiateur vibre au réel comme son public le ferait s'il était la et comme il le fait quand il est la sur les lieux.

Le repli de l'État national laisse un vide dans lequel s'engouffrent aussi bien le dépit des électeurs face aux politiciens (L'actualité, 1995), la nostalgie de la norme et de l'autorité exprimée par les intégrismes (Le Devoir, 1995) que la glorification des différences jusqu’à l'individualisme et l'émiettement. Le déverrouillage tous azimuts qu'il provoque signifie pour les journalistes et les communicateurs, le transfert de la clé de voute de leur grille de fabrication du sens du politique à l'existentiel.

Pour le journaliste, cette recentration s'accompagne d'un alignement tout aussi radical sur l'entreprise qui l'emploie et sur la sensibilité du public sectoriel qu'elle dessert, plutôt que sur l'idée abstraite du public. Pour les communicateurs, les institutions publiques se faisant plus modestes et se retirant d'elles-mêmes dans l'impuissance, il reste le soutien aux activités commerciales plus immédiates. Dans une mesure plus grande qu'avant, on s'attend aussi à ce qu'ils interviennent auprès de l'opinion publique sectorielle susceptible d'influencer négativement les activités économiques de l'employeur par la construction d'une image moins positive, par le colportage de rumeurs et de critiques ou par la traduction du mécontentement en actions de boycottage sous différentes formes plus ou moins directes.

Les uns et les autres sont emportés par la logique de séduction qui contraint tout le monde sur la scène publique. En matière politique, l'attrait pour la conduite des affaires d'un Etat, affaibli à tous points de vue et proclamant chaque jour son désir de modestie, a beaucoup diminué. Cependant, même lorsqu'il ne s'agit plus de conquérir ou d'influencer directement l'État, le simple fait pour un groupe de s'inscrire dans une majorité constitue déjà une possibilité d'influence horizontale sur tous. Les médias sont le lieu principal du déploiement de charme de chaque groupe en quête de telles alliances.

En bref, l'éclairage du postmodernisme conduit à conclure que le professionnel de l'information et de la communication, comme les autres intellectuels, est dépouillé de son rôle de "législateur de la connaissance et des valeurs culturelles " à côté des autres prêtres de la Raison et du progrès. D'en-haut, ils avaient le droit et la légitimité d'interpréter le monde au profit de la masse. La menace qui aujourd'hui pèse sur tous ces spécialistes des « humanités » se nomme l'obsolescence. On les somme de « légitimer leur pertinence dans des sociétés technocratiques où les sciences refoulent les autres disciplines » (Best et Kellner, 297). 

leur expertise technique en matière de rhétorique et de séduction, pour laquelle la demande augmente d'autant plus que ces activités sont devenues une règle du jeu fondamentale de la démocratie fonctionnelle.

\section{BIBLIOGRAPHIE}

\section{RÉFÉRENCES}

ALBERT, M. Capitalisme contre capitalisme. Paris : Seuil, 1991.

BARTHA, Peter La gestion des questions d'intérêt public. Gestion. vol. 15. № 4. Novembre 1990, p. 62-69.

BUSINESS WEEK, What happened to Advertising ?, September 23, 1991, pp. 66-72

BEST, Steven and Douglas KELLNER Postmodern Theory-Critical Interrogations. New York : The Guilford Press, 1991, 324 pages

BOISVERT, Yves Le Postmodernisme. Montréal : Boréal, 1995,124 pages

CANCLINI, Nestor Garcia et Rafael RONCAGLIORO - editores, Cultura transnational y culturas populares. Lima : Instituto para America Latina (IPAL), 1988, 375 pages

CHARRON, Jean, Les médias et les sources : les limites du modèle de l'agenda-setting. Hermès 17-18. Paris : CNRS Editions, 1995, P. 73-92

CHARRON, Jean, «Les médias, les sources et la production de l'information ». Charron, Jean, Jacques Lemieux et Florian Sauvageau - dir. (1991). Les journalistes, les médias et leurs sources. Boucherville : gaëtan morin éditeur, 1991,p. 171-204

COULON, Jocelyn, La revanche de Dieu - Le fanatisme religieux menace toutes les sociétés, estime un spécialiste de l'islam. Le Devoir. Mardi 28 novembre, 1995, p. 1-10

DACHEUX, Éric, Les stratégies de communication persuasive dans l'Union européenne. Paris :

L'Harmattan, 1994, 286 pages de Bonville, Jean, La Presse québécoise de 1884 à 1914 genèse d'un média de masse. Québec : Les Presses de l'Université Laval, 1988, 416 pages

DELLI Carpini, MICHAEL X. et Bruce A. WILLIAMS, Methods, Metaphors, and Media Research: The Uses of Television in Political Conversation. Communication Research. vol. 21, $\mathrm{n}^{\circ}$ 6. December 1994, p. $782-812$

DEMERS François, « Journalistic ethics : The rise of the 'good employee's model' : a threat for professionalism? », The Canadian Journal of Communication, 4 (2), May 1989, p. 15-27

DEMERS, Francois, Du côté des journalistes et de « leurs » messages : le cas de l'information et de la publicité dans les années 80, p. 215-242 in Beauchamp, Michel (direction). Communication publique et société - repères pour la réflexion et l'action. Boucherville : gaëtan morin éditeur, 1991, 403 pages 
DEMERS, François, Crise de l'État-nation et communication Politique interne : une ligne de fracture nommée démocratisation. Hermès 17-18. Paris : CNRS Éditions, 1995, p. 267-285

DICKENS, David R. et Andrea FONTANA, ed. Postmodernism and Social Inquiry. New York : The Guilford Press, 1994, 259 pages

ELLUL, Jacques, l'information et le système technicien. Propagandes. Paris ; Economica, 1990, p. 343-352 (Texte paru aussi dans la Revue française de Communication 1976, n 1, p 7-16)

Fédération professionnelle des journalistes (FPJQ). Des nouvelles sans relations de presse, est-ce possible? Thème du congrès annuel de décembre 1988. Journal du congrès. 12 pages

GRUNIG, James E. \& Todd HUNT (1984). Managing Public Relations, New York : Holt, Rinehart and Winston.

HALIMI, Serge, Course à l'audience et aux recettes publicitaires - Les médias américains délaissent le monde. Le Monde diplomatique. $\mathrm{N}^{\circ}$ 485. 41 ${ }^{\mathrm{e}}$ année. Août 1994, p. 28

JURGENSEN, Karen et Philip MEYER, After Journalism. Journalism Quartely. vol. 69, n² 2. Summer 1992, p. 266-272

L'actualité, Politiciens - Crise de confiance. Les citoyens décrochent. Massivement. Enquête sur un phénomène sans précédent. Montréal. Décembre. 1995, vol. 20, N 19, p. 25-31

LAPONCE, Jean Antoine, « Pour ne pas conclure - De la pyramide à l'éteignoir : la grande peur des masses », p. 211-221, in ZYLBERBERG Jacques (dir.), Masses et postmodernité, Québec, Les Presses de l'Université Laval, Paris, Méridiens Klincksieck, 1986, 248 pages

LAVOINNE, Yves, « Le Journaliste, l'Histoire et l'historien -Les avatars d'une identité professionnelle (1935-1991) », Réseaux - Communication Technologie Société, CNET, n 51, Janvierfévrier 1992 , p. 39-53

LAVOINNE, Yves, «Les métamorphoses de l'information », Études de communication 15, 1994, p. $90-110$

Maffesoli, Michel, Au creux des apparences - pour une éthique de l'esthétique. Paris : Plon, 1990, 316 pages

MCLUHAN, Marshall, D'œil à oreille. Montréal : Hurtubise HMH, 1977, 166 pages

MURDOCK, Graham, Technologie, économie et pratique journalistique : une perspective socio-historique. Demain, l'informateur - Du transcripteur de dépêches à l'agent de novation. Bruxelles : éditions JEB, 1982, p. 83-104

NOËL, André, Journalistes-Relationnistes : pas de promiscuité. Le ‘30’, 1988, vol. 12. n 9. p. 6

PICHETTE, Jean, « La nouvelle utopie démocratique », Relations, Montréal, 1994, n 605, novembre : p. 267-271

RUELLAND, Denis, «Le professionalisme du flou », in Réseaux - Communication Technologie Société, CNET, n 51, janvier-février 1992, p. 25-37

RUELLAND, Denis, Le professionalisme du flou - Identité et savoir-faire des journalistes francais, Presses universitaires de Grenoble, 1993, 240 pages

THUOT, Jean-François, Déclin de l'État et formes postmodernes de la démocratie. Revue québécoise de science politique. $\mathrm{N}^{\circ} 26$. Automne, 1994, p. 75-102

TRACEY, Michael and Wendy W. REDAL, The New Parochialism : The Triumph of the Populist in the Flow of International Television. Canadian Journal of Communication. vol. 20. $\mathrm{N}^{\circ}$ 3. Summer 1995, p. $343-365$ 
TREMBLAY, Gaëtan et LACROIX, Jean-Guy. Télévision -Deuxième dynastie. Sillery, Presses de l'Université du Québec, 1991,163 pages

WASKO, Janet and Vincent MOSCO, Editors, Democratic Communications in the information age. Toronto : Garamond Press, 1992, 249 pages.

WOLTON, Dominique, Le journalisme, victime de son succès, Médiaspouvoirs, $\mathrm{n}^{\circ} 13$, janvier-févriermars 1989, p. 51-62.

\section{RÉSUMÉS}

La communication gagne l'information et le sens critique qui fondait le journalisme lui fait de plus en plus cruellement défaut. C'est le développement de l'information existentielle liée aux études marketing. Mais c'est aussi l'inscription dans une logique politique "postmoderniste »: l'avènement d'une démocratie fonctionnelle. Le déclin de l'Etat-nation et de la culture fragilise tous les pouvoirs, en particulier le « quatrième pouvoir », celui de la presse.

Communication is gaining ground in information and the critical sense which is the basis of journalism is more and more absent. This is the development of existential information linked to market studies. But it is also involved in a political postmodern logic : the arrival of a functional democracy. The decline of the nation-state and of culture have weakened all powers and in particular that of the " Fourth Estate ", the Press.

\section{AUTEUR}

\section{FRANÇOIS DEMERS}

François Demers a été journaliste professionnel, principalement dans des quotidiens mais aussi à la télévision, de 1965 à 1980. Détenteur d'un diplôme de philosophie de l'Université Laval, il y enseigne le journalisme depuis 15 ans. Il est l'auteur de deux livres et co-directeur de deux autres. Il a aussi publié une vingtaine d'articles scientifiques et chapitres de livres. Ses champs d'intérêt principaux sont les changements technologiques, l'éthique et la rhétorique journalistique. Depuis 1987, M. Demers est doyen de la Faculté des Arts de l'Université Laval de Québec. 